\section{P1-79 ASSOCIATION BETWEEN CHANGES IN ANTHROPOMETRIC MEASURES THROUGH ADOLESCENCE AND CARDIOVASCULAR RISK FACTORS AT 17 YEARS OF AGE}

doi:10.1136/jech.2011.142976c.72

1,2,3 J Araujo, 1,2,3E Ramos. ${ }^{1}$ Department of Hygiene and Epidemiology, University of Porto Medical School, Porto, Portugal; ${ }^{2}$ Department of Cardiovascular Research \& Development Unit, University of Porto Medical School, Porto, Portugal; ${ }^{3}$ Institute of Public Health, University of Porto, Porto, Portugal

Objective To study the association of changes in body mass index (BMI) and waist circumference (WC) with cardiovascular risk factors in late adolescence.

Methods As part of a population-based cohort (EPITeen) 1574 adolescents were evaluated when they were $13 \mathrm{y}$ and $17 \mathrm{y}$. We computed OR and $95 \%$ CIs using logistic regression to study the association between changes in BMI z-scores and WC from 13 to 17 years and outcomes at 17 years. The outcomes were define as high systolic blood pressure $(\mathrm{SBP}) \geq 130 \mathrm{~mm} \mathrm{Hg}$; high diastolic blood pressure $(\mathrm{DBP}) \geq 85 \mathrm{~mm} \mathrm{Hg}$; low high density lipoprotein cholesterol (HDLc) if first quartile and high: triglycerides, low density lipoprotein cholesterol (LDLc), glucose and insulin if fourth quartile. Results Comparing with adolescents who remained normal weight in both study waves, those who remained overweight/obese presented higher odds of high SBP [females: $\mathrm{OR}=3.33$ (1.78-6.23); males: $\mathrm{OR}=3.17$ (2.00-5.01)], DBP [females: $\mathrm{OR}=2.47(1.00-6.10)$; males: $\mathrm{OR}=2.37$ (1.17-4.79)], triglycerides [males: $\mathrm{OR}=3.25$ (1.88-5.62)], LDLc [males: $\mathrm{OR}=3.50(2.03-6.00)]$, insulin [females: $\mathrm{OR}=2.18$ (1.26-3.79); males: $\mathrm{OR}=2.32(1.34-4.03)]$ and low HDLc [females: $\mathrm{OR}=2.21$ (1.28-3.80); males: $\mathrm{OR}=1.94$ (1.11-3.41)]. The estimates for those who changed to overweight/obese were similar. Among those who changed to normal weight, we found associations in the opposite direction, although statistical significance was not achieved. Regarding changes in WC and considering the 75th percentile as cut-off, results were similar, however, partly explained by BMI. Conclusion Remaining or changing to higher anthropometric measures in adolescence period was associated with worse cardiovascular risk profile later in adolescence.

\section{P1-80 DIFFERENT EFFECTS OF BODY MASS INDEX AND PHYSICAL ACTIVITY ON THE RISK OF ANKLE, WRIST AND HIP FRACTURES IN POSTMENOPAUSAL WOMEN}

doi:10.1136/jech.2011.142976c.73

\begin{abstract}
1,2M Armstrong, ${ }^{* 1,2}$ E Spencer, ${ }^{1,2} \mathrm{~B}$ Cairns, ${ }^{1,2} \mathrm{~V}$ Beral. ${ }^{1}$ University of Oxford, Oxford, Oxfordshire, UK; ${ }^{2}$ on behalf of the Million Women Study collaborators, Oxford, Oxfordshire, UK
\end{abstract}

Introduction We compared the relationship between body mass index (BMI) and physical activity for the risk of ankle, wrist and hip fractures in a large prospective study of postmenopausal women in the UK.

Methods In 1996-2001, women recruited into the Million Women Study completed a self-administered questionnaire asking about a range of health and lifestyle factors. Incident fractures were identified through self-report in a follow-up questionnaire completed on average 3.2 years after recruitment. RRs and CIs for each fracture site in women by BMI and physical activity at recruitment were calculated using Cox regression models, adjusted for socioeconomic status, and other factors.

Results Among 599648 postmenopausal women, there were 5117 ankle fractures, 8564 wrist fractures, and 753 hip fractures. When compared to lean women $\left(\mathrm{BMI}<20.0 \mathrm{~kg} / \mathrm{m}^{2}\right)$, obese women $\left(B M I \geq 30 \mathrm{~kg} / \mathrm{m}^{2}\right)$ had an increased risk of ankle fracture $(\mathrm{RR}=2.47$; $95 \%$ CI 2.32 to 2.63$)$, but a decreased risk of wrist fracture $(R R=0.69$; $95 \%$ CI 0.65 to 0.73 ) and hip fracture ( $R R=0.29 ; 95 \%$ CI 0.23 to
0.37). Physical activity had little influence on the risk of ankle or wrist fracture, but women who reported partaking in strenuous physical activities were at a lower risk of hip fracture than women who reported being never/rarely active ( $R R=0.65 ; 95 \%$ CI 0.56 to 0.76 ). Conclusion BMI and physical activity have different effects on the incidence of fracture at different sites. While obese women are at increased risk of ankle fracture they are at lower risk of wrist and hip fracture. Physical activity has no marked influence on ankle and wrist fracture but is protective against hip fracture.

\section{P1-81 HIV, HSV-2 AND SYPHILIS AMONG MARRIED COUPLES IN INDIA: PATTERNS OF DISCORDANCE AND CONCORDANCE}

doi:10.1136/jech.2011.142976c.74

${ }^{1} \mathrm{P}$ Arora, ${ }^{* 2,3} \mathrm{~N}$ Nagelkerke, ${ }^{1,4} \mathrm{~S}$ K Sgaier, ${ }^{5} \mathrm{R}$ Kumar, ${ }^{6} \mathrm{~N}$ Dhingra, ${ }^{1} \mathrm{P}$ Jha. ${ }^{1}$ Centre for Global Health Research (CGHR), St. Michael's Hospital, Dalla Lana School of Public Health, University of Toronto, Toronto, Ontario, Canada; ${ }^{2}$ Department of Community Medicine, United Arab Emirates University, Al-Ain, United Arab Emirates; ${ }^{3}$ Department of Public Health, Erasmus Medical Center, Rotterdam, The Netherlands; ${ }^{4}$ The Bill \& Melinda Gates Foundation, New Delhi, India; ${ }^{5}$ School of Public Health, Postgraduate Institute of Medical Education and Research, Chandigarh, India; ${ }^{6}$ National AIDS Control Organization, Ministry of Health \& Family Welfare, Government of India, New Delhi, India

Introduction Differences in sexual networks likely explain the disparity in the scale of HIV epidemics in sub-Saharan Africa and India. HIV and sexually transmitted infection (STI) discordant couple studies provide insights into important aspects of these sexual networks. We wished to quantify the role of male sexual behaviour in HIV transmission in married couples.

Methods We analysed patterns of HIV, HSV-2 and syphilis seroconcordance and discordance in married couples from two community surveys in India: the National Family Health Study-3 for HIV-1 (a nationally representative household survey) and the Centre for Global Health Research health check-up for HSV-2 and syphilis. A statistical model was used to estimate the fraction of infections introduced by each of the two partners accounting for higher mortality and separation among HIV concordant couples.

Results Only $0.8 \%, 16.0 \%$ and $3.5 \%$ of couples were infected with HIV-1, HSV-2, and syphilis, respectively. A large proportion of infected couples were discordant $(73.1 \%, 55.0 \%$ and $84.2 \%$ for HIV1, HSV-2, and syphilis, respectively). Among couples with any STI, the male partner introduced the infection the majority of the time (HIV-1: 85\%, HSV-2: 62\%, syphilis: 75\%).

Conclusions Male infidelity appears to be the driving force of the HIV/STI epidemic our study population and likely in the Indian population at large. Ensuring safe male client and female sex worker contacts should remain a primary target of the National AIDS Control Program in India.

\section{P1-82 PREVALENCE AND CORRELATES OF HERPES SIMPLEX VIRUS-2 AND SYPHILIS INFECTIONS IN THE GENERAL POPULATION IN INDIA}

doi:10.1136/jech.2011.142976c.75

${ }^{1,2} \mathrm{~S}$ K Sgaier, ${ }^{1,3} \mathrm{P}$ Mony, ${ }^{3} \mathrm{~S}$ Jayakumar, ${ }^{1} \mathrm{C}$ McLaughlin, ${ }^{1} \mathrm{P}$ Arora, ${ }^{*} \mathrm{R}$ Kumar, ${ }^{5} \mathrm{P}$ Bhatia, ${ }^{1} \mathrm{P}$ Jha. ${ }^{1}$ Centre for Global Health Research, Li Ka Shing Knowledge Institute, St. Michael's Hospital, Dalla Lana School of Public Health, University of Toronto, Toronto, Ontario, Canada; ${ }^{2}$ Bill and Melinda Gates Foundation, New Delhi, India; ${ }^{3}$ St John's Research Institute, St John's National Academy of Health Sciences, Bangalore, Karnataka, India; ${ }^{4}$ School of Public Health, Post Graduate Institute of Medical Education and Research, Chandigarh, India; ${ }^{5}$ Indian Institute of Health \& Family Welfare, Hyderabad, Andhra Pradesh, India

Introduction To determine the prevalence and correlates of Herpes Simplex Virus-2 (HSV-2) and syphilis infections in the general population in India. 